\title{
Nanotechnology offers alternative ways to fight COVID-19 pandemic with antivirals
}

\author{
A new wave of funding focuses on antiviral nanomaterials as pandemic countermeasures.
}

A flurry of recent papers highlights growing interest in approaches that employ nanomaterials as antiviral countermeasures. Compared with traditional small molecules or antibodies that inhibit viral replication or cellular entry, nanotechnology offers drug developers virus binders, cell-membrane decoys or viral-envelope inhibitors that may complement conventional antiviral therapies. With the help of an influx of funding spurred by the COVID-19 pandemic, some researchers hope that these materials could soon move towards clinical translation.

Nanomaterials have already played a key role in the fight against SARS-CoV-2. The Pfizer-BioNtech and Moderna vaccines both rely on lipid nanoparticles to carry mRNA into cells. Nanoparticles are also showing promise as vehicles for small-molecule antiviral drugs, building on decades of progress with nanoscale drug-delivery systems.

Now the urgency of the COVID-19 pandemic is generating interest in therapeutic nanomaterials that can themselves halt viruses in their tracks, rather than just acting as delivery vehicles for drugs or vaccines. "A lot of these nanomaterials are being developed to engage with the virus particles directly, either disrupting them or binding to them," says Joshua A. Jackman of Sungkyunkwan University in South Korea.

Unlike traditional therapeutics, which tend to target a specific viral species and may lose their efficacy as the virus accumulates mutations, antiviral nanomaterials target chemical and physical properties common to many types of virus. Several recent papers have described antiviral strategies that rely on

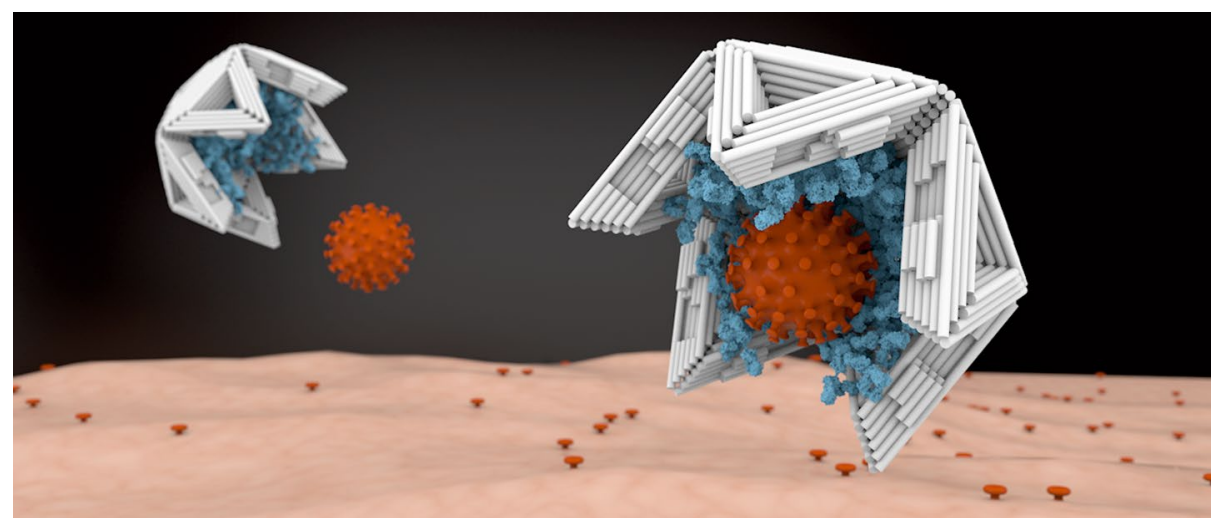

In this artist's impression, a DNA shell traps a virus to stop it from interacting with host cells. Credit: Elena-Marie Willner / Dietz Lab / TUM

DNA-based nanostructures to trap viruses or use modified polymers that act as cell membrane decoys; others break apart viral membranes to prevent infection. Some of these nanomaterials may offer advantages in the context of pandemic countermeasures, as they can potentially be formulated quickly and have activity across a broad range of virus families.

Much of this work is still confined to academic labs, although a handful of companies are developing antiviral nanomaterials (Table 1). But the ravages of COVID-19-and the clear need to prepare for future viral pandemics-are opening up fresh opportunities.

In June, for example, the Biden administration launched the Antiviral Program for Pandemics, with $\$ 3$ billion for research into new antivirals that can tackle SARS-CoV-2 and other viruses with pandemic potential. "This new funding stream will definitely stimulate and support more research and development in the area of antiviral nanomaterials," says Liangfang Zhang at the University of California, San Diego. "COVID has really changed the landscape, we see that we really need more ready-made solutions for emerging viruses."

Because many viruses rely on glycoproteins on their surface to bind to molecules on host cells, nanomaterials that mimic these cellular attachment points can potentially act as antivirals. Zhang is making 'nanosponges' that use this approach to intercept viruses. To make the nanosponges, Zhang's team starts with human cells such as red blood cells or macrophages. After removing the contents of the cell to leave only the membrane, they break the membrane into thousands of tiny vesicles roughly 100 nanometers wide. Then they add nanoparticles made from a biocompatible and biodegradable polymer, such as poly(lactic-co-glycolic acid). Each nanoparticle becomes coated with a cell

Table 1 | Selected companies pursuing antiviral nanomaterials

\begin{tabular}{llll} 
Company & Technology & Target examples & Progress \\
\hline Atom Bioworks & DNA stars & Dengue, SARS-CoV-2 & Preclinical \\
Cellics Therapeutics & Nanosponge cell membrane decoys & SARS-CoV-2, dengue, pneumonia & Preclinical \\
Starpharma & Dendrimers & HIV, herpes simplex virus, SARS-CoV-2 & $\begin{array}{l}\text { Marketed products include VivaGel (in condoms) } \\
\text { and Viraleze antiviral nasal spray }\end{array}$ \\
NanoViricides & Nanoviricide micelles & $\begin{array}{l}\text { Shingles (varicella zoster virus), herpes } \\
\text { simplex virus, dengue, HIV, SARS-CoV-2 }\end{array}$ & Preclinical
\end{tabular}


membrane, forming a stable core-shell structure that acts as a decoy of a human cell. The nanosponges then use binding points on their membranes to surround a virus and prevent it from entering host cells.

These nanosponges are effective against a range of viruses and bacteria in vivo, and Zhang's San Diego-based spin-out company Cellics Therapeutics plans to begin a clinical trial next year of its lead candidate, a nanosponge carrying a red blood cell membrane that is effective against methicillin-resistant Staphylococcus aureus (MRSA) pneumonia. Cellics is also using macrophage membranes to develop similar nanosponges with antiviral activity. "There are many different types of virus, and each virus may have different variants," Zhang says, "but regardless of that, in order to infect humans they need to interact with the host's cells through receptors."

Last year, Zhang found that a cellular nanosponge coated in membranes derived from human lung epithelial type II cells or human macrophages were both able to trap SARS-CoV-2 and prevent infection in vitro. The membranes on these nanosponges sport angiotensin-converting enzyme 2 (ACE2) and CD147, to which SARS-CoV-2 binds during infection. Zhang's team also has unpublished results from an in vivo study with mice, showing efficacy against the coronavirus and no evidence of toxicity.

Starpharma, headquartered in Abbotsford, Melbourne, Australia, is also mimicking host cells to combat viruses. It makes synthetic polymers with a branched structure, known as dendrimers, that are roughly 3-4 nanometers wide. The outer surface of each dendrimer is covered in naphthalene disulfonate groups, similar to the heparan sulfate proteoglycans found on host cell membranes, which many viruses stick to.

Starpharma already has products on the market that employ a dendrimer called SPL7013 as an external barrier against viruses and bacteria. SPL7013 is used in VivaGel, a lubricant in condoms, for example. Earlier this year, Starpharma launched Viraleze, a broad-spectrum antiviral nasal spray containing SPL7013, which is registered for sale as a medical device in Europe and India. However, sales of Viraleze in the UK were halted in June after the UK Medicines and Healthcare products Regulatory Agency raised concerns about the product's marketing claims.

In August, the company unveiled research showing that Viraleze prevented SARS-CoV-2 infection in a mouse model. Administering the nasal spray before and after exposure to SARS-CoV-2 reduced viral loads in the animals' blood, lungs and trachea by more than $99 \%$. The company says that a clinical safety study, which has not yet been peer reviewed, showed that the dendrimer in Viraleze was not absorbed in the body and caused no significant side effects.

Jackie Fairley, Starpharma's CEO, says that the company's dendrimer could prove useful in future pandemics. "It's a stable raw material that could be formulated into a product quickly, and it has activity across a very broad spectrum of viruses," she says. In the meantime, the company plans to carry out larger animals studies to confirm the activity of Viraleze against SARS-CoV-2.

Some antiviral nanomaterials are precisely shaped to trap viruses. In Germany, Rainer Haag of the Free University of Berlin is covering silica nanoparticles with 5-10-nm-tall spikes that mesh neatly between a virus' surface glycoproteins. The spikes can be decorated with sialic acid sugars to enhance binding, or with antiviral compounds such as zanamivir. "By matching the morphology of the virus, we maximize the binding," says Chuanxiong $\mathrm{Nie}$, a postdoc in Haag's group who has been leading the work. In vitro experiments showed that the particles prevented infection of cells with influenza A virus, and the team now hopes to design spiky nanoparticles with activity against SARS-CoV-2. The Berlin University Alliance is supporting the work as part of a $€ 1.8$-million ( $\$ 2.3$ million) grant awarded last year.

Star-shaped DNA scaffolds offer another potential approach. Xing Wang at the University of Illinois at Urbana-Champaign has built such structures carrying DNA aptamers capable of binding to antigens at multiple points on the surface of dengue fever virus. The physical bulk of the DNA star, and its negative charge, prevent the virus from latching on to host cells, shutting down infection. The team also has in vitro data, currently being peer reviewed, showing that certain DNA stars can inhibit SARS-CoV-2 infection. Wang aims to commercialize the DNA stars through his spin-out company, Atom Bioworks of Cary, North Carolina.

A form of DNA origami is being pursued by Hendrik Dietz at the Technical University of Munich. The team has developed shells made from DNA that are large enough to swallow an entire virus whole. The interior of the self-assembling icosahedral shells can be lined with binders, such as antibodies, to hold onto trapped viruses. Dietz says that the nanoshells could potentially decrease viral load during acute infections.

The researchers designed triangular DNA structures that assemble into shells of various shapes and sizes, from 90 to

\section{Roche deal with RNA-editing spinout}
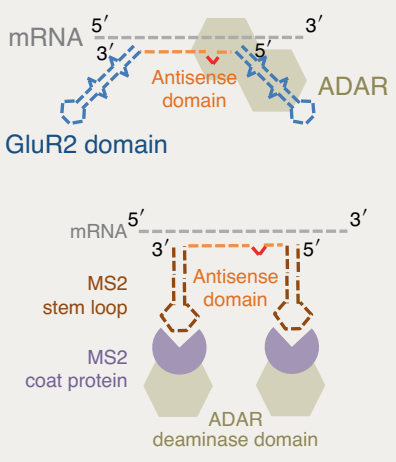

In vivo RNA editing of point mutations via RNA-guided adenosine deaminases. Reprinted with permission from Katrekar, D. et al. Nat. Methods 16, 239-242 (2019), Springer Nature.

Shape Therapeutics has announced a \$3-billion multi-target collaboration with Roche. The two-year-old Seattle-based biotech is developing a platform for designing and delivering base editors of RNA in vivo. Their system, developed by co-founder Prashant Mali of UCSD's Department of Bioengineering, recruits endogenous adenosine deaminase (ADAR) via a small guide RNA (gRNA) comprising glutamate receptor 2 (GLUR2) domains linked to an RNA-targeting antisense oligo. Their approach avoids the potential delivery and immunogenicity problems associated with heterologous ADAR and the genotoxicities associated with conventional DNA-editing enzymes. In 2019, Mali's groups published a proof-of-concept article in which they demonstrated in vivo RNA editing in mouse models of muscular dystrophy and ornithine transcarbamylase deficiency.

The collaboration will center around Alzheimer's disease, Parkinson's disease and rare diseases. Shape will do the preclinical work of identifying gRNAs against RNA targets and creating adeno-associated virus (AAV) variants through DNA synthesis and mutational analysis.

Roche acquired Spark Therapeutics (which markets gene therapy Luxturna for retinal diseases) and forged deals with Sarepta (gene therapy for muscular dystrophy) and Dyno (AAV capsid design by machine learning).

Published online: 7 October 2021 https://doi.org/10.1038/s41587-021-01097-x 


\section{UK trials first CRISPR- edited wheat in Europe}

In a sign of the United Kingdom's intentions to move away from European Union (EU) restrictions following Brexit, its Department for Environment, Food \& Rural Affairs (DEFRA) has given scientists at Harpenden-based Rothamsted Research the go-ahead for the first field trials of gene-edited wheat made using CRISPR technology anywhere in the UK. The wheat has reduced amounts of the naturally occurring amino acid asparagine, which converts to the potentially carcinogenic chemical acrylamide when bread is baked or toasted. By knocking out the asparagine synthetase gene TaASN2, researchers have demonstrated more than a $90 \%$ reduction of asparagine concentrations in the grain of one line of edited wheat. The trials are expected to run for five years, measuring the amount of asparagine in the same wheat grain when grown in the field, as well as assessing other aspects such as yield and protein content. The aim is to produce ultra-low-asparagine, non-gene-modified wheat, according project leader Nigel Halford. Current EU regulations treat gene editing of crops the same as genetic modification, though in April the European Commission launched a review of its rules on genetically modified organisms, paving the way to a possible loosening of restrictions for plants resulting from gene-editing technology. DEFRA secretary George Eustice said at a farming conference last January that the UK, outside of the EU, was now free to make its own decisions based on the science, calling the current EU approachenshrined in a 2018 European Court of Justice ruling - "flawed and stifling to scientific progress."

Published online: 7 October 2021 https://doi.org/10.1038/s41587-021-01098-w
300 nanometers wide. By tweaking the DNA sequences in the triangular building blocks, they created virus-sized openings in the side of a shell. In vitro experiments showed that these shells could bind viruses such as adeno-associated virus serotype 2 and prevent them from infecting human cells. "The advantage of our shells is the number of virus binders we can attach, and also that we can switch the virus binders very easily," says Christian Sigl, a PhD student in Dietz's lab who carried out the experimental work. This means the shells could in principle be tailored to bind any virus, he says. Dietz is the coordinator of a $€ 3$.9-million project called Virofight, which launched in June 2020 with funding from the European Commission, to build a shell to trap SARS-CoV-2 and test the strategy in mice.

Some nanomaterials go beyond simply binding viruses-instead, they disrupt the viral membrane to prevent infection. Viral genomes are encapsulated by a protein-based capsid, but in many cases, including that of SARS-CoV-2, that capsid is covered by a phospholipid bilayer membrane, which is essential for the virus to fuse with cell membranes. Unlike bacterial membranes, this viral envelope is acquired from the host cell membrane itself, as newly created virus particles leave infected cells. "This envelope is critical for infection, and for the structural integrity of the virus," Jackman says. "But people don't necessarily realize the lipid membrane is druggable."

NanoViricides, based in Shelton, Connecticut, aims to disrupt viral membranes using soluble polymer surfactants that form spherical micelles. These nanoviricide structures are decorated with up to 1,200 ligands, such as peptides, that bind to viral glycoproteins. The micelles then fuse with the viral membrane, damaging it so that it can no longer infect a host cell.

The company had been gearing up for a clinical trial of a topical nanoviricide for treating shingles, but last year it pivoted to focus on COVID-19. In March, it publicized positive results from in vivo studies of two nanoviricides against SARS-CoV-2. In addition to its usual mechanism of viral membrane disruption, one of the nanoviricides carried the antiviral molecule remdesivir in its core. Both nanoviricides significantly extended survival times in rats with lethal coronavirus lung infections as compared with remdesivir treatment alone.
Although these study results have not yet been peer reviewed, the company says it is preparing to take the two nanoviricides into clinical trials.

Jackman is also developing antiviral peptides, which slot into the viral membrane and aggregate to form pores. "Once a critical number of holes is formed in a membrane, it's kind of like Swiss cheese, and it just collapses," says Jackman, who has used this strategy to successfully treat lethal Zika virus in mice.

For now it's still early days for all of these technologies. "It's a very niche area, but I think it's growing. And there is definitely interest in it," says Kathie Seley-Radtke, a medicinal chemist at the University of Maryland, Baltimore County, who develops small-molecule antiviral agents and is president-elect of the International Society for Antiviral Research. "The bottom line is, we can't discard any possibility right now, because COVID is so serious."

Jackman adds that pharma and biotech companies are generally taking a cautious approach to therapeutic nanomaterials. There are still concerns about bioaccumulation of the nanoparticles, for example, and potential long-term side effects. But he says the recent progress with lipid nanoparticles in mRNA vaccines clearly shows that nanomaterials can be useful in tackling viruses, which may bolster confidence.

Another barrier is that many of the in vivo studies on these materials have used a diverse range of protocols, making them difficult to compare. Some protocols involve preincubating the antiviral nanomaterial with the virus before administering the mix to an animal, or giving the antiviral to the animal before exposure to a virus. To help more nanomaterials move into clinical trials, Jackman suggests that researchers need to agree on standardized animal models and performance benchmarks, and focus on evaluating antiviral nanomaterials in animals that are infected with a virus first. "The material science is just awesome for all these concepts," Jackman says. "I think that the next frontier is to really make this more translational."

\section{Mark Peplow}

Penrith, UK

Published online: 7 October 2021

https://doi.org/10.1038/s41587-021-01085-1 